\title{
Milliliter per Kilogram per Hour
}

National Cancer Institute

\section{Source}

National Cancer Institute. Milliliter per Kilogram per Hour. NCI Thesaurus. Code C73759.

A dose calculation unit expressed in milliliter(s) per kilogram per period of time equal to sixty minutes. 